\title{
EFFECTIVE APPLIED VOLTAGE AS AN INDICATOR OF THE RADIATION EMITTED BY AN X-RAY TUBE
}

\author{
By Lauriston S. Taylor, G. Singer, and C. F. Stoneburner
}

\section{ABSTRACT}

A previous study ${ }^{1}$ suggested a parallelism between the applied effective voltage and the emission of an X-ray tube. The investigation has been carried further under more carefully controlled conditions. It is found for two mechanical rectifiers and one "constant potential" generator that the X-ray emission per effective (root-mean-square) milliampere of tube current is about the same for all generators operating at the same effective (r. m. s.) voltage (the radiation passing through the same filter in all cases). Thus it becomes possible to express all radiations in terms of that excited by constant potential. This leads to a simplification in the description and reproduction of irradiation conditions in biological work. Although the study has been limited to three generators, it is believed that they are sufficiently typical to warrant generalization.

\section{CONTENTS}

I. Introduction

II. Apparatus

1. Current measurements

2. Voltage measurement́s_..............................

3. Radiation measurements

III. Experimental results

IV. Discussion $\ldots \ldots \ldots$

\section{INTRODUCTION}

In a recent study the energy emitted by an X-ray tube, as measured by an ionization chamber, was found to be very different when excited by different types of high voltage generators furnishing the same average tube current and peak voltage (as measured by a sphere gap). But it was further found that for the same average currents and the same effective voltage ${ }^{2}$ of the generators, the emission, as measured by the ionization chamber, is approximately the same.

The total energy in an X-ray spectrum devoid of characteristic lines varies as the square of the instantaneous applied voltage, and hence for a known voltage wave form the total energy and its wavelength distribution, for the period of a complete cycle, is calculable. Because of the presence of tungsten line radiation about $70 \mathrm{kv}$, it is not yet practicable to predict the total X-ray energy by integrating over one cycle of the voltage wave. In the present work the X-ray emission per unit tube current, as it varies with peak voltage, with average voltage, and with effective voltage per unit tube current is given for more carefully controlled and wider ranges of conditions. This is expressed in terms of the X-ray emission per milliampere. 


\section{APPARATUS}

Thus far three generators have been used, designated by $A, B$, and $C$ as in the preceding work. ${ }^{3} A$ is a full wave mechanical rectifier having a single high tension transformer, and rectifying over approximately 30 degrees of cycle; $B$ is also a full wave mechanical rectifier but with a divided high tension transformer (two transformers in a single tank), and rectifying over approximately 20 degrees of the cycle; $C$ is a valve-tube-condenser ripple potential ${ }^{4}$ generator (so-called "constant potential") having a ripplage of about 2 per cent per milliampere. The three generators could be interchangeably conconnected to the same X-ray tube.

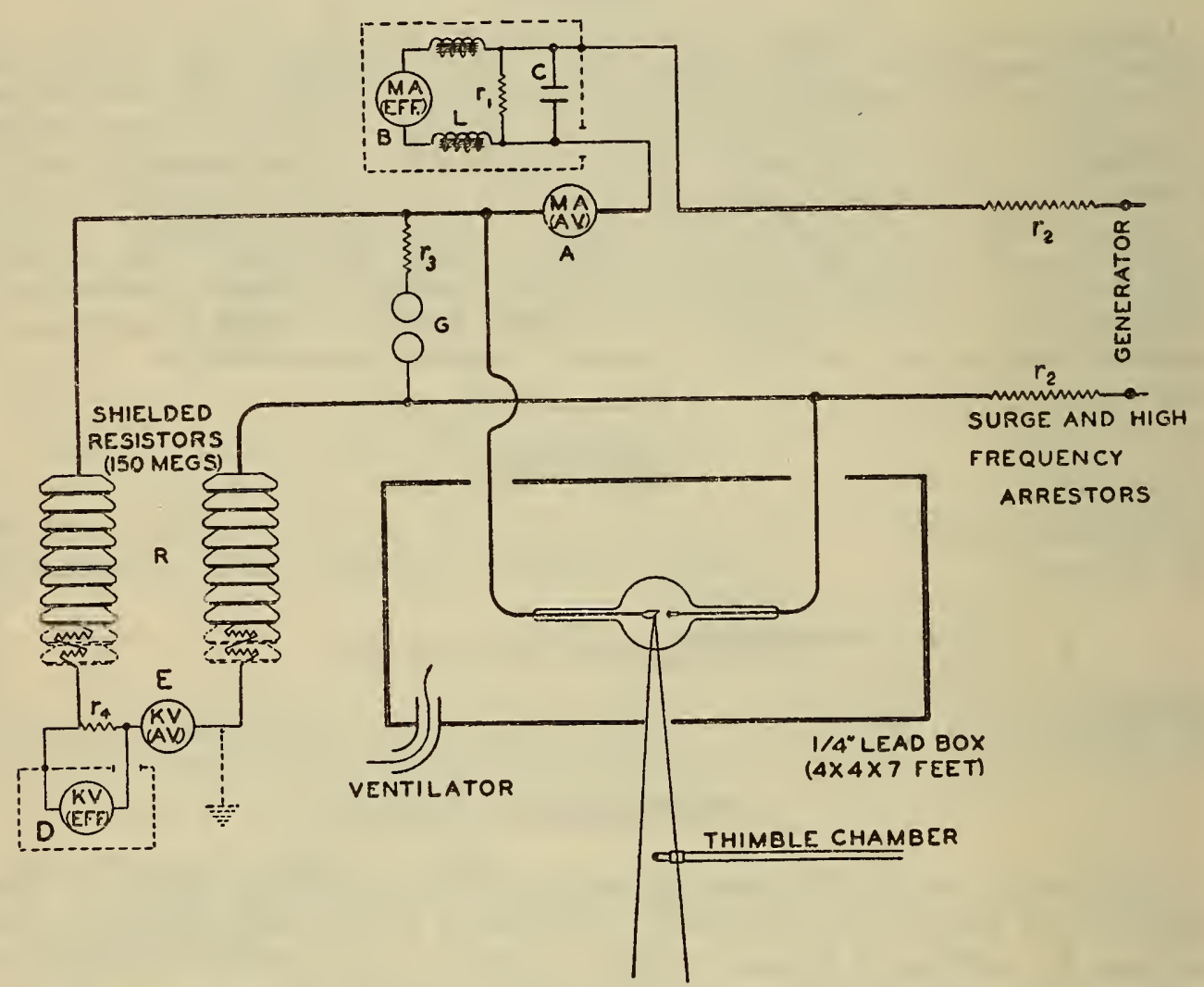

Figurw 1.-Apparatus showing voltage and current measuring equipment

\section{CURRENT MEASUREMENTS}

The arrangement of the apparatus is indicated in Figure 1. Average current through the tube is measured in the usual manner by a d.c. milliammeter $A$; and the effective current by an a.c. milliammeter, $B$. For this purpose a thermo milliammeter alone was available. This was protected from high-frequency oscillations by iron core chokes, $L$, having a d. c. resistance of a fraction of an ohm; a noninductive resistor, $r$, of about $10,000 \mathrm{ohms}$, and a $1 / 20 \mu \mathrm{f}$ capacitor, $C$.

\footnotetext{
3 See footnote 1, p. 561 .

4 The term "constant potential" has been used loosely to describe the potential supplied by kenotron of other valve tube rectification. A more accurate designation of a voltage which is not constant in fact but fluctuates slightly about a certain average value is "ripple voltage." "Thus by a "ripple quantity" (potential or current) is meant 2 simple periodic quantity $y=V_{0}+V_{1} \sin \left(\omega x+\alpha_{1}\right)+V_{2} \sin \left(2 \omega x+\alpha_{2}\right)+---$ in which the constant term $\left(V_{0}\right)$ is so large that all values of the quantity are positive (or negative). The amount of ripple ("ripplage" or "ripplance") in a ripple quantity is the ratio of the difference between the maximum and minimum values of the quantity to the average value. These definitions are under consideration by the Committee on Electrical Definitions of the American Standards Association under the sponsorship of the A. I, E, E.
} 
Since the resistance of the meter, $B$, was only about 20 ohms, this shunt system served as a very effective filter against high-frequency oscillations in the high-tension system, without affecting the sensitivity.

Excessive high-frequency oscillation when using the mechanical rectifiers caused difficulty in peak-voltage measurements with the sphere gap, but this was largely eliminated by placing two $1 / 2$ megohm inductively wound surge resistors, $r_{2}$, immediately next to the rectifier switches, giving smoother operation of the equipment and more consistent peak voltage measurements. After raising the generator voltage sufficiently to compensate for the potential drop across the resistors, it was found that their presence did not alter the tube output in any way.

With generator $C$, the same resistors were used for the purpose of limiting the current in case of slight tube gassing or when using a sphere gap for measuring the voltage. It was found that they raised very materially the voltage limit at which a given tube would operate satisfactorily.

\section{VOLTAGE MEASUREMENTS}

Peak voltage of all three generators was measured by the gap between $12.5 \mathrm{~cm}$ spheres, protected by a water resistance $r_{3}$, of the order of 5 megohms to prevent heavy arcing at the gap and consequent tripping of circuit breakers.

Average voltage was measured by means of a d. c. microameter, $E$, in a series with a shielded high voltage resistor of 150 mehohms, ${ }^{5}$ the meter being at the center of the resistance so as to be at or near ground potential. ${ }^{6}$

Effective voltage was measured by means of a 150 -volt calibrated Kelvin multicellular electrostatic voltmeter, $D$, shunted across a resistance, $r_{4}$, of $75,000 \mathrm{ohms}$. Since its potential was likely to be different from ground, it was necessary to shield it by a metal case having the same potential as one of its terminals.

It should be stated here that the insertion of the high-voltage resistor, $R$, across the line has no noticeable influence upon the operation of the high-tension generators because the current it shunts off is very small. This fact is of course essential, since a wave form very different from that under normal operating conditions may otherwise result.

\section{RADIATION MEASUREMENTS}

The X-ray emission was measured by means of a thimble chamber, kept in a fixed position with respect to the tube throughout the study. The beam was in all cases filtered by $0.525 \mathrm{~mm}$ of copper, the wall effect of the chamber being negligible for the transmitted radiation.

No other determination of radiation quality was undertaken, as the previous study ${ }^{7}$ indicated what was to be expected under the operating conditions employed here, namely, all beams having the same effective voltage and filtration and giving equal intensities have approximately the same quality (whether expressed in the halfvalue layer of copper, copper absorption coefficient, or effective wave length).

5 L. S. Taylor, B. S. Jour. Research, yol. 5 (RP217), p. 609, 1930.

6 The voltage on the high-tension conductors is seldom divided equally above and below ground potential, although the error in measurement introduced by grounding the resistor at the center is very small.

7 L. S. Taylor and K. L. Tucker. See footnote 1, p. 561 . 


\section{EXPERIMENTAL RESULTS}

In this study, to eliminate the effect of variation in operation, simultaneous values were obtained for (1) average current, (2) effective current, (3) peak voltage, (4) average voltage, (5) effective voltage, and (6) X-ray tube output for a constant filteration of $0.525 \mathrm{~mm}$ of copper, the average current (the quantity measured in practice) serving as independent variable.

In Figure 2 , groups $A$ and $B$ show the tube emission, expressed in terms of the ionization current per average milliampere ( $\mathrm{I} / \mathrm{ma}$ (average)), as a function of peak voltage for generators $A$ and $B$, respectively, and for different tube currents, $2,3,4$, and 5 ma. With generator $B$ it is seen that for a given peak voltage, the value of $I / m a$ (average) varies from 10 to 15 per cent in changing the operating

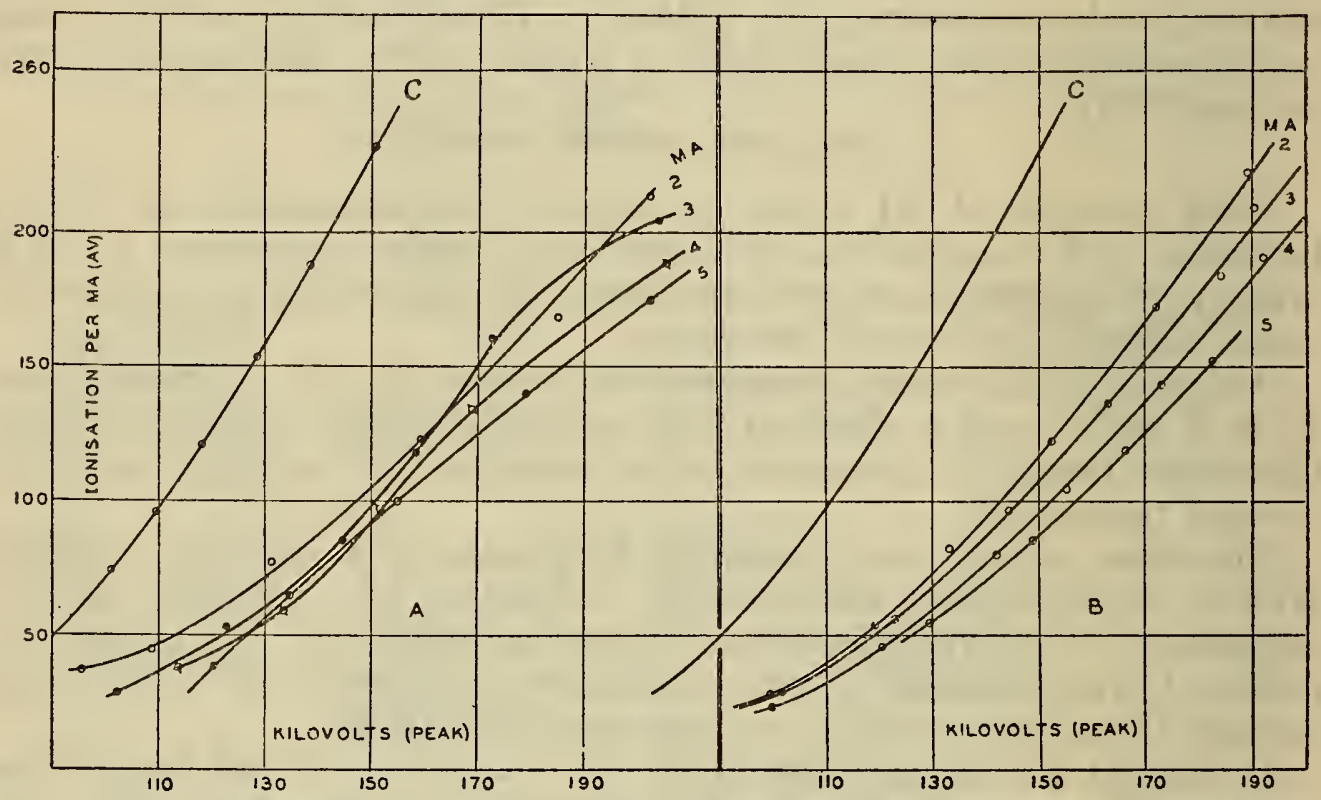

Figure 2.-Relationship between $X$-ray tube emission per average milliampere, and the applied peak voltage

$A, B$, mechanical rectifiers; $C$, constant potential.

tube current by $1 \mathrm{~mA}$. This is not unexpected since the generator wave form depends to a large extent upon the power drawn from the generator. It is obvious from these curves that it may be very misleading to attempt to deduce the emission of an X-ray tube from current and peak voltages alone. For generator $A, \mathrm{I} / \mathrm{ma}$ (average) varies erratically with peak voltage under different operating currents.

Figure 2, curve $C$ in both groups is from generator $C$ ("constant potential") for a tube current of $4 \mathrm{ma}$. Since the peak voltage of this generator does not vary appreciably with tube current (average voltage being kept constant), a single curve embodies the results. It is seen that for the same peak voltage there is a large difference between the X-ray emission of generator $C$ (curve $C$ ) on the one hand and that of generators $A$ and $B$ (curves $A$ and $B$ ) on the other. To obtain the same X-ray emission from generators $A$ and $B$ as from generator $C$, the peak voltage of $A$ and $B$ must be very much larger than that of $C$, depending upon the operating current. 
If, for the same radiations plotted in Figure 2, we plot instead the ionization per average milliampere against the average voltage, corresponding curves, $A, B$, and $C$ in Figure 3 are obtained. It will be noted that I/ma (average) again varies with the tube current. In the case of generator $A$ the values of $I / \mathrm{ma}$ (average) vary from about 2 to 8 per cent in changing the operating current by 1 ma. This percentage change becomes smaller, however, in going to higher average voltages, whereas, when the potential is measured in peak kilovolts, the changes become larger at higher voltages.

In the case of generator $B$ the same general results obtain. The variation of $I / \mathrm{ma}$ (average) with tube current at a given average voltage is, however, much greater at lower potential than for $A$ while at higher potentials it is about the same.

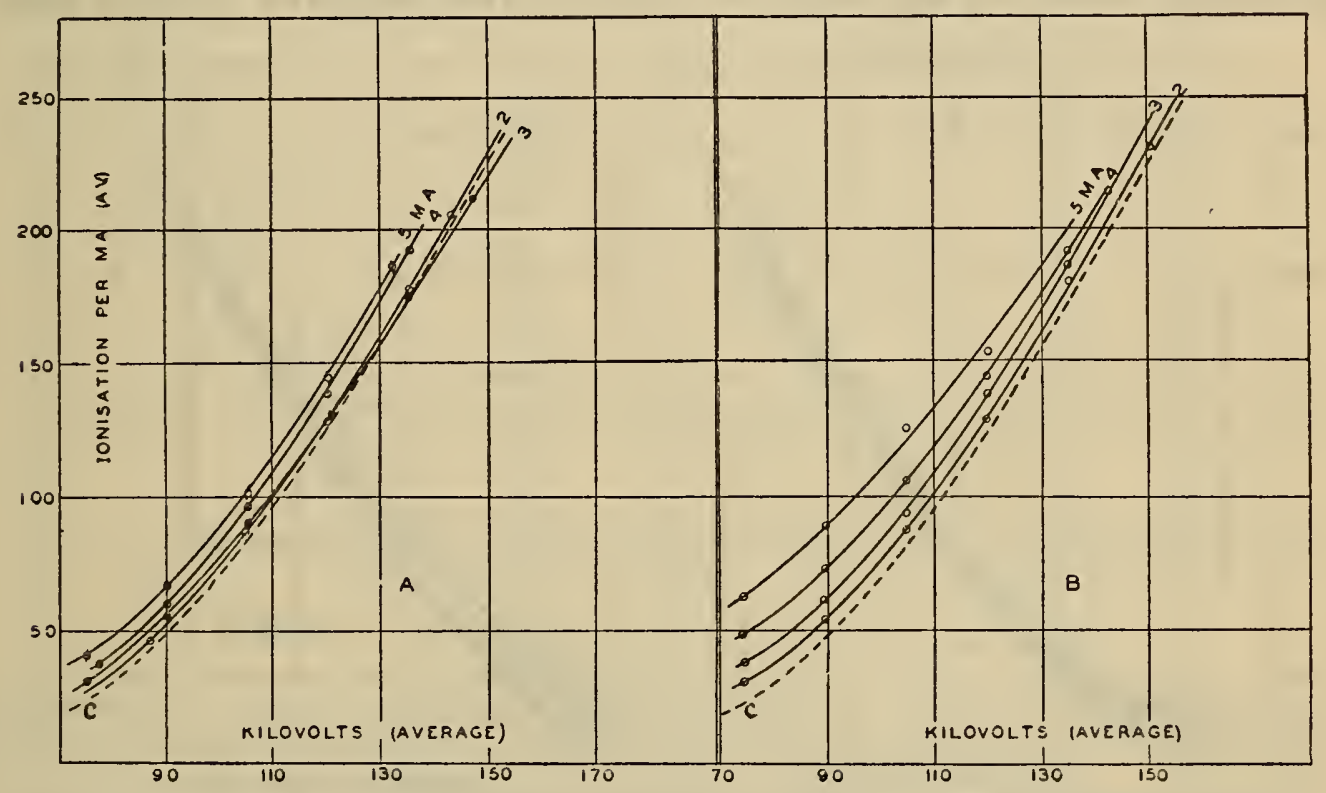

Figure 3.-Relationship between $X$-ray tube emission per average milliampere, and the applied average voltage

$A, B$, mechanical rectifiers; $C$, constant potential.

It is seen that, while the curves for $A$ and $B$ differ with tube current, they all lie in the region of curve $C$ (dashed) as contrasted to the corresponding curves of Figure 2 for peak voltages. Thus, if the current and voltage for all three generators be measured in terms of average milliamperes and average kilovolts (that is, by using d. c. instruments), the tube emission per milliampere is in all cases of the same order of magnitude.

Using effective milliamperes and effective kilovolts, curves for generators $A, B$, and $C$ for the $\mathrm{X}$-ray emission per effective milliampere are given in Figure 4.

It should be pointed out that for the comparatively small ripplage present in the voltage of generator $C$, the difference between the average and effective voltage is so small that it may be neglected. Likewise their variation with tube current is negligible; hence, a single curve for $I / \mathrm{ma}$ (effective) gives a satisfactory basis of comparison.

In the case of generator $A$ the change in emission per milliampere, as the operating current is varied, is present and somewhat erratic as 
in the case of the peak voltage curves. The percentage change, however, is the least of the three cases given. Similarly in the case of generator $B$ the change is present, but again to the least degree.

Of prime importance, however, is the fact that the curves (fig. 4) for both generators $A$ and $B$ lie very closely along the curve for constant potential-much more closely at the higher voltages than those in Figure 3 for average current and voltage measurements. It is concluded that, for equal effective X-ray tube currents and equal effective voltages, the emission per milliampere will be sufficiently near the same with all three generators, for the effective current and voltage to be used as characteristic of the X-ray emission.

Since the emission per milliampere for all strictly constant potential generators is the same, it is logical that it be referred to as a base. By means, then, of the effective current and effective voltage mea-

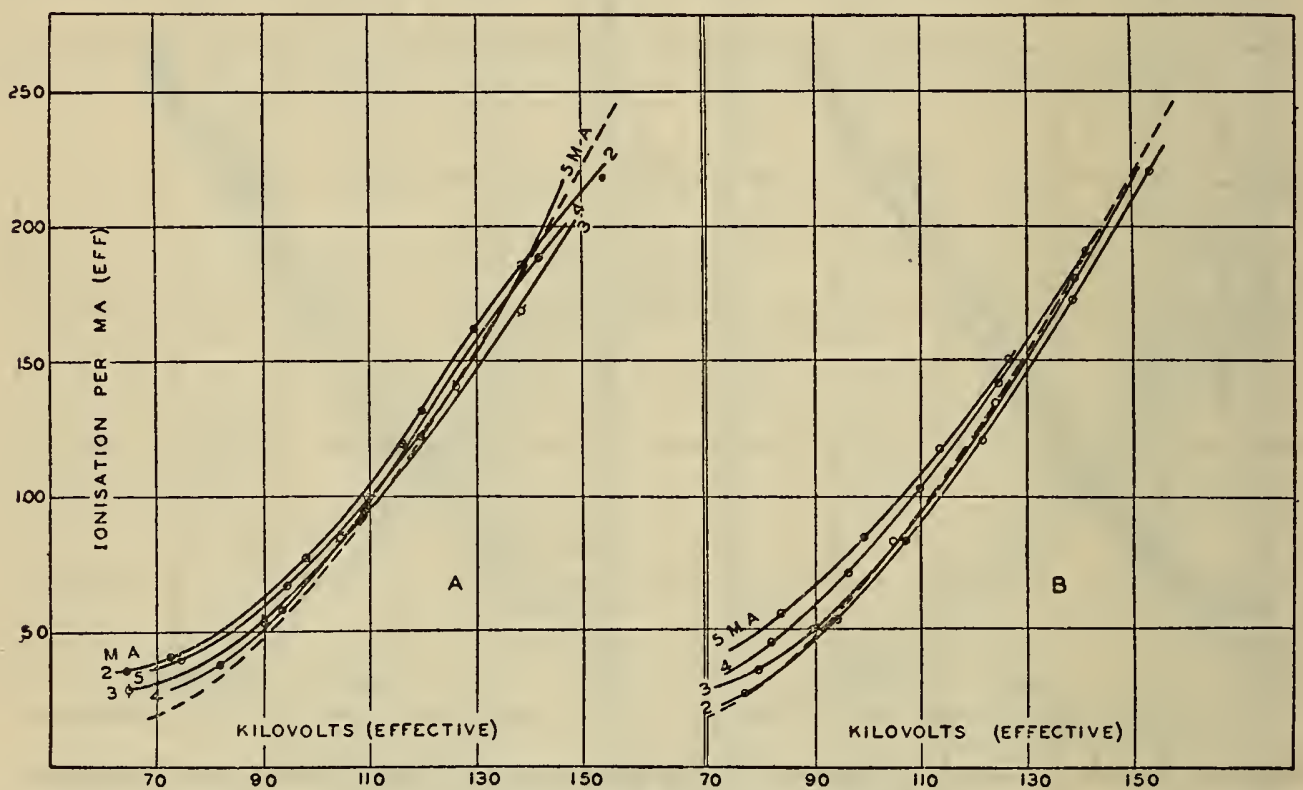

FigURE 4.-Relationship between $X$-ray tube emission per effective milliampere, and the applied effective voltage

$A, B$, mechanical rectifiers; broken line curve, constant potential.

surements described, it is possible to relate consistently the X-ray emission excited by the various types of generators.

It is possible to trace the residual variation in X-ray emission per milliampere at lower voltages with the operating current, largely to the current-voltage characteristics of the X-ray tube. This will account also for the divergence from the constant potential base line. Figure 5 shows, for various applied constant potentials, the tube current curves for three values of the filament current, $I_{f}$. It is seen that, since the tube current is still increasing about 5 per cent per 30 kilovolts at 120 kilovolts, saturation is not attained. Consequently, with the application of a varying voltage to the tube, the effective current is bound to depend upon the voltage wave form to a varying degree.

This is illustrated in Figure 6 where curves $a$ and $b$ are two bypothetical tube current characteristics, the second of which is ideal in that it reaches saturation at a comparatively low voltage. Curves $D, E$, and $F$ represent three types of generator voltage wave form 
having the same effective voltage, $e$, which, when applied to tubes having characteristics $a$ and $b$, produce the tube current wave forms $a^{\prime}$ and $b^{\prime}$, respectively, for each associated voltage wave. In each of

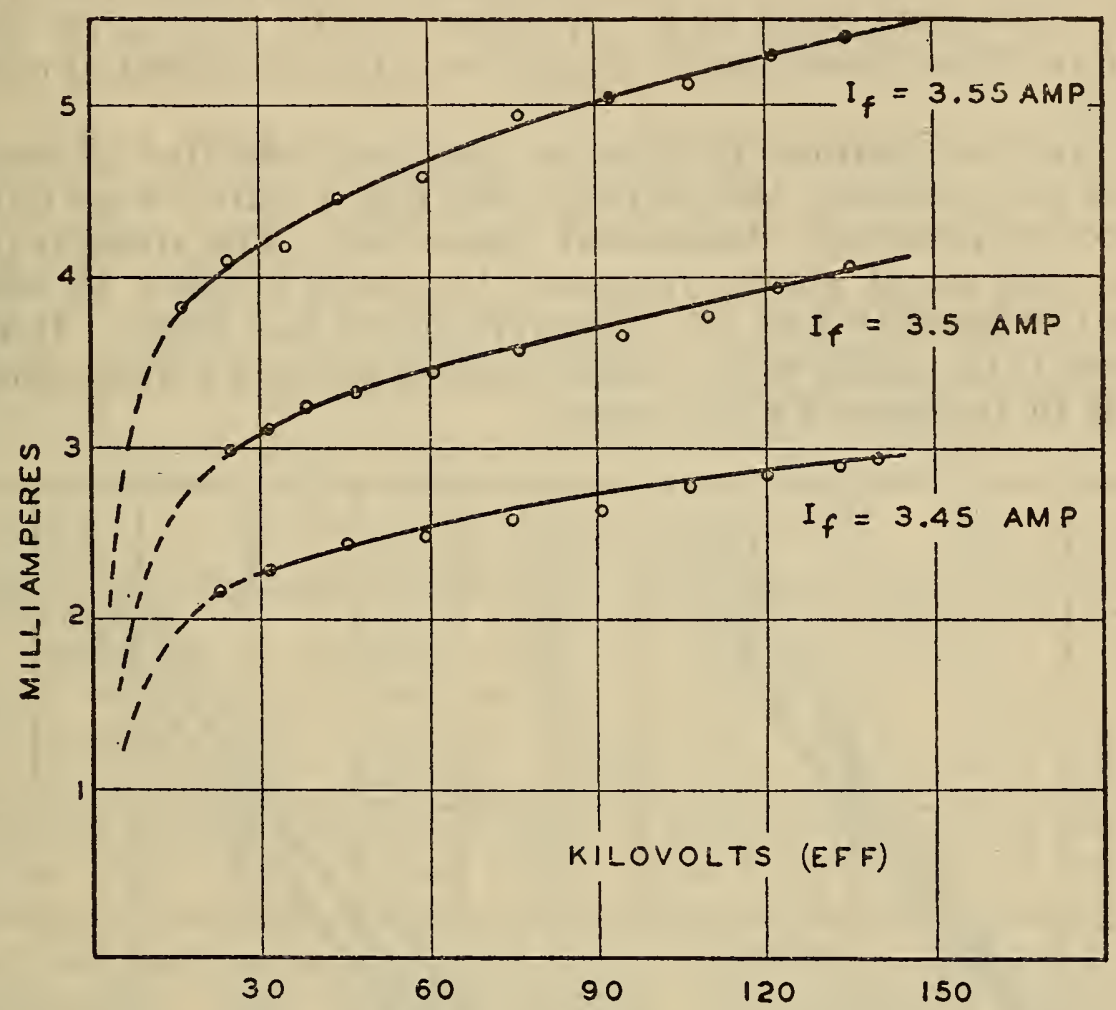

Figdre 5.-Tube current-tube voltage characteristics

the cases it is seen that the effecitve current $i_{a}$ for tube characteristic $a$ is lower than the effective current $i_{b}$ for characteristic $b$. Moreover, the magnitude of this difference depends upon the generator voltage wave form as shown.

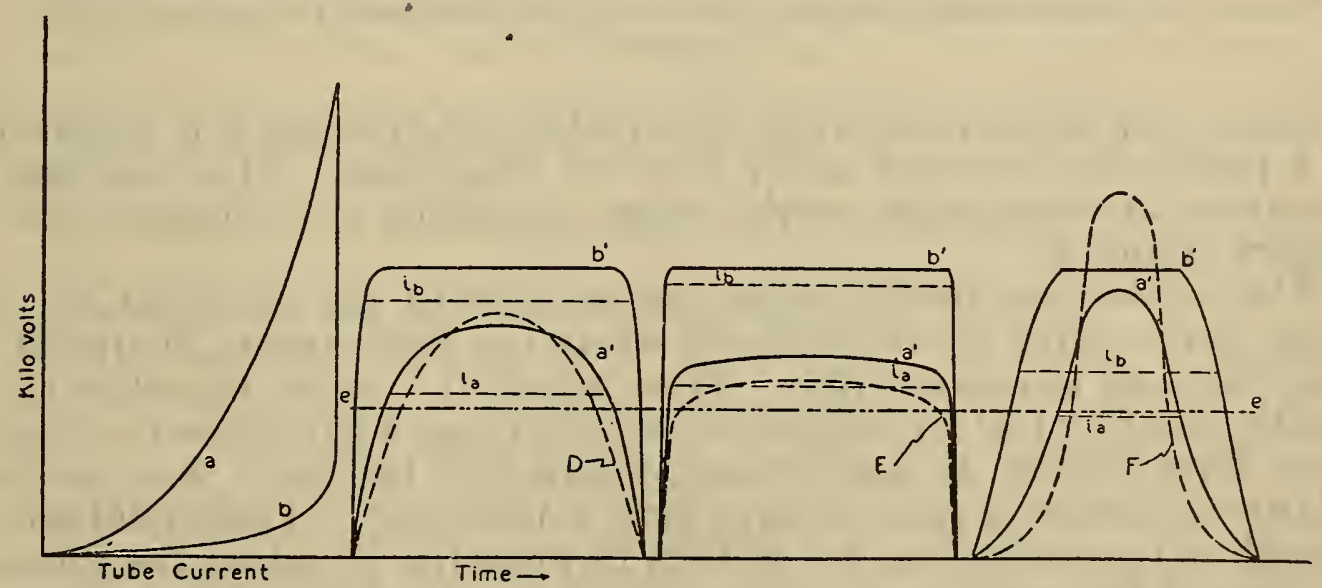

FIgURE 6.-Effect of tube characteristic and generator wave form on the effective tube current

To compare the explanation above with the results found, we note that the experimentally determined tube characteristics in Figure 5 are similar to $a$ in Figure 6; hence, the tube current measured in 
effective amperes is less than it would have been had the tube reached saturation at as low voltage as for characteristic $b$.

In Figure 4 the emission per effective milliampere-which is plotted against effective kilovolts - would accordingly have been less with an ideal characteristic such as $b$. In other words, the curves plotted would have fallen more nearly along the constant potential curve $C$. (Fig. 4.)

To show the relationship between peak and effective kilovolts for different tube currents, the curves $A$ and $B$ in Figure 7 were obtained for the correspondingly designated generators. The straight dashed lines in each set of curves represent the ratio of peak to effective voltage $(\mathrm{kv}($ peak $)=1.41 \mathrm{kv}$ (effective)) for a sine wave. It is seen that there is no simple relationship between the two voltage measurements as in the case of a sine wave.

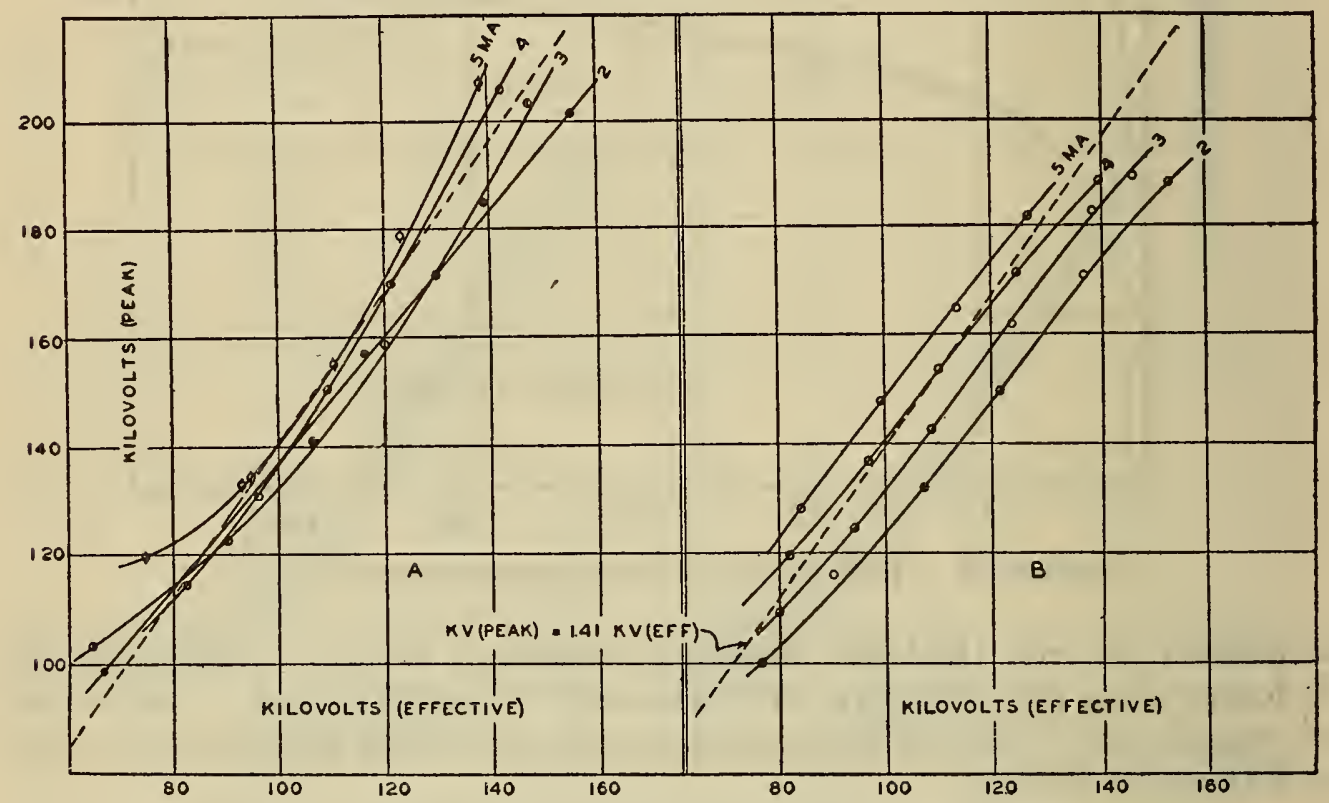

FigURE 7.-Relationship between peak and effective kilovolts for two mechanical rectifiers

Such a set of curves is very useful when interpreting the behavior of a particular generator under different conditions. This was demonstrated in some earlier work ${ }^{8}$ when comparing the output of generators $A$ and $B$.

The curves also furnish some inference as to the generator wave form, particularly above the point where the tube current characteristic plays an important rôle. Thus, where the points lie below the line $\mathrm{kv}$ (peak) $=1.41 \mathrm{kv}$ (effective), they indicate a flat-topped voltage wave form relative to a sine wave; where they lie above that line, a relatively narrow or peaked wave form is indicated. These relations, though only qualitative, are of help in properly adjusting the phase position of the switches on a mechanical rectifier for optimum X-ray tube emission.

8 See Figure 16 with explanation in paper by L. S. Taylor and K. L. Tucker. (See footnote 1, p. 561.) 


\section{DISCUSSION}

There has been considerable confusion in interpreting the physical magnitudes involved in the therapeutic use of high-voltage $X$ rays. The greatest single factor contributing to this confusion has been the voltage measurement, since it has been sometimes assumed that a given X-ray tube, activated by the same voltage (by sphere gap measurement) and passing equal average currents, should yield the same X-ray emission for all similar generators. We have shown here, however, that the emission may differ by \pm 20 per cent under supposedly like conditions.

Although a difference existing between mechanical and constant potential rectifiers has been generally recognized, there has been uncertainty as to any exact relationship between them, with the result that few attempts have been made to correlate treatment factors for the two machines. There has been even a belief that the radiation of one could not be clinically reproduced by the other. The experiments described here show that both generators may be made to produce the same emission (as far as may be deduced from absorption measurements in copper or paraffin) when the tube potentials are properly adjusted.

The present study has been limited thus far to mechanical and "constant potential" rectifiers. While it is believed by some that mechanical rectifiers will probably be displaced by valve-tube rectifiers, the study is of no less value, since with the great variety of valve-tube circuits in use there is an equal variety of voltage wave forms produced, none of which may be readily correlated as far as they affect the X-ray output of a tube. For example, in addition to "constant potential" there will be half-wave, full-wave, and polyphase rectifiers and voltage doubling circuits, such as the La Tour and Villard systems. In some of these the wave form depends upon load to a greater extent than in others and, as a consequence, measurements of peak voltage alone will lead to similar discordance now encountered in mechanical rectifiers.

This study reveals that, when the generator potential is measured in effective kilovolts, the X-ray emission of a tube is equivalent to that produced by very nearly the same constant potential. It is thus possible to express the emission in terms of that obtainable from a constant potential source.

Expressing tube potential in effective kilovolts simplifies the method of expressing radiation quality. For example, when describing radiation quality in terms of the "half-value layer of copper" it is recognized as necessary to also state the voltage. However, as voltages are now measured, even this is inadequate due to the effect of wave form. To correctly indicate quality by the usual absorption methods, the effective voltage should be stated - not the peak voltage.

The cordial cooperation of the Radiological Research Institute and of the American X-ray equipment manufacturers in this work has been very helpful.

Washington, June 17, 1932. 